\title{
A Preliminary Study to Identify Data Needs for Improving Fit of Hand and Wrist Orthosis Using Verbal Protocol Analysis
}

\author{
Xinyang Tan ${ }^{1}$, Wei Chen ${ }^{2}$, Jiangang $\mathrm{Cao}^{2}$ and Saeema Ahmed-Kristensen ${ }^{3}$
}

1. Dyson School of Design Engineering, Imperial College London, London SW7 2DB, UK

2. The Affiliated Xuzhou Rehabilitation Hospital of Xuzhou Medical University, Xuzhou City 221009, China

3. INDEX, Dept of Science, Innovation, Technology, Entrepreneurship, University of Exeter, London SE1 8ND, UK 


\title{
A Preliminary Study to Identify Data Needs for Improving Fit of Hand and Wrist Orthosis Using Verbal Protocol Analysis
}

\author{
Xinyang $\operatorname{Tan}^{1}$, Wei Chen ${ }^{2}$, Jiangang $\mathrm{Cao}^{2}$ and Saeema Ahmed-Kristensen ${ }^{3}$
}

\begin{abstract}
The delayed delivery, poor fitting and discomfort of customized orthoses are reported in rehabilitation clinics as resulting in more invasive interventions. The current practice of orthosis customization relies heavily upon the experience and fabrication processes of therapists. In order to better understand the current practice, and thus identify data that is required for better comfort moving towards a data-driven customization, this paper describes a study generating working models of therapists. Customizations of hand and wrist orthoses for 18 patients were observed. Verbal protocol analysis was employed to extend the current understanding of fabrication processes. Working models of four therapists were established with quantitative evaluation on major phases, interactive activities and iterations of performing tasks during fabrication, revealing different working models between in- and out-patient departments (e.g. fabrication for in-patients was more complex and focused on ergonomic fitting whereas fabrication for out-patients paid attention to durability) which were qualitatively explained.
\end{abstract}

\section{Keywords: task analysis; verbal protocol analysis; comfort; working model; orthosis}

Practitioner Summary : Fit and comfort are imperative for orthosis design and fabrication, however the current practice of customization of an orthosis relies upon the experience of individual hand therapist. The paper presents working models of hand therapists, and relevant data that would enable customization of orthosis for better fit.

Word count: 5634

\section{INTRODUCTION}

$\mathbf{R}$ ISING demand and variation in delivery and quality of customized orthoses prescribed to patients have been revealed in a report of the National Healthcare System for England [1]. The need to improve the orthotic services in terms of low patient compliance during fabrication and using orthoses have also been highlighted. Low patient compliance has been found due to side effects, including discomfort [2], [3], [4] and pressure sores [5], [6], [7], caused by poor fit of orthoses. A more efficient customization is in need to fit orthosis "right first time", which prevents more expensive and invasive interventions like surgery [8], [9]. As the main purpose of an orthosis is to fit and stabilize body segments, and tasks conducted by hand therapists (referred to as therapists in this paper) during customizations are pre-defined to achieve the goal, a better fit can be accomplished by improving the efficiency of relevant tasks or phases during a fabrication process. However, there are challenges that inhibit the improvement. First, orthosis fabrication is a craft-based operation, i.e. highly dependent on experience and skills of individual therapist [10], which leads to variance of fabrication processes. Thus, a standard method to improve the fabrication may not fit in customization of all therapists. Second, since the fabrication process is an implicit and complex system involving multiple phases with application of various tools and techniques in different clinical contexts, it is difficult to clearly identify the critical phase to be modified. Therefore, there is a need to understand the current fabrication process, and the factors that influence the customization. This will enable the possibility of identifying techniques to improve fit, for instance, defining correct dimensions [11], [12], disclosing excessive contact pressure [13] and selecting proper materials [14]. Without this understanding, the approach to improve fit of orthoses is not efficient, as the customization varies in clinics and relevant data that informs customization is unknown and thus orthosis customization will remain bespoke.

Principles were proposed in literatures to guide the customization involving various fitting techniques. For example. Fess et al. [15] highlighted five aspects regarding the hand orthosis fabrication, including mechanics, mobilization assists, design, construction and fit. A seven-step fabrication process in an organized manner, including how to evaluate fit while molding, was presented in [16] for handy reference of therapists. The study by Richards et al. [8] provided a guidance for front-line staff on the

This work was partially supported by the Affiliated Xuzhou Rehabilitation Hospital of Xuzhou Medical University, the Xuzhou Central Hospital, the China Scholarship Council and the Imperial College London (Corresponding author: Xinyang Tan).

${ }^{1} \mathrm{X}$. Tan is with the Dyson School of Design Engineering, Imperial College London, London SW7 2DB, UK

xinyang.tan14@imperial.ac.uk

${ }^{2}$ W. Chen and J. Cao are with the Affiliated Xuzhou Rehabilitation Hospital of Xuzhou Medical University, Xuzhou City 221009, China $189521728460163 . \mathrm{com}$

${ }^{3}$ S. Ahmed-Kristensen is with INDEX, Dept of Science, Innovation, Technology, Entrepreneurship, University of Exeter, London SE1 8ND, UK s.ahmed-kristensen@exeter.ac.uk 


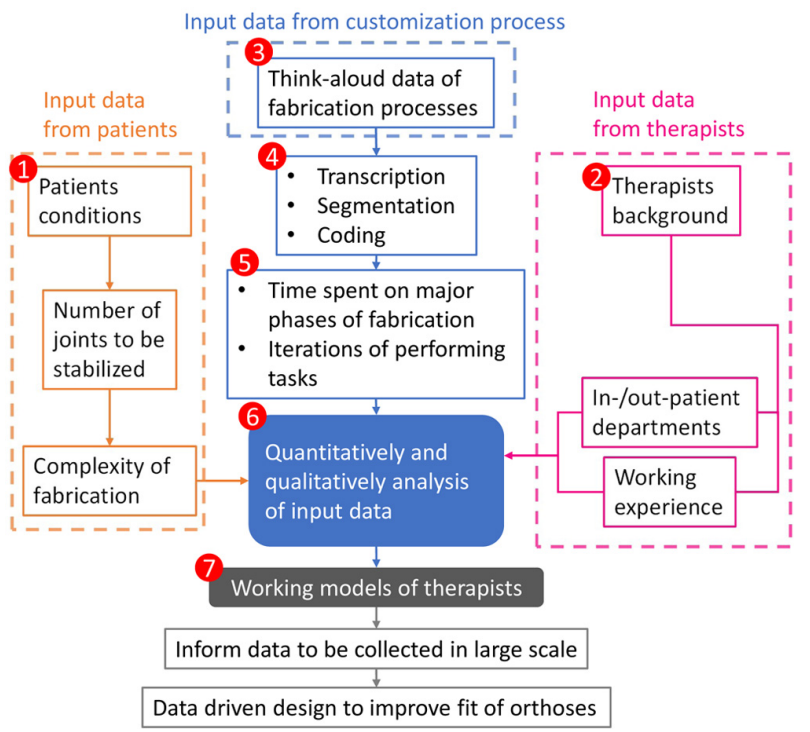

Fig. 1: The method of deriving working models of therapists.

Fig. 1 Alt Text: A workflow diagram with three branches regarding data collection from three perspectives, i.e. patients, therapists and the customization process. The three branches are centralized at the step (6) demonstrated in Section II and followed by a single workflow with step (7) and further steps detailed in Section III.

management of hand fractures, highlighting appropriate fabrication techniques to have better fit with anatomical considerations. Though criteria of fabrication have been demonstrated in these studies, following previously established principles, therapists tend to build their own skills and working habits through clinical practice, since fabrication occurs in different contexts with different clinical needs, for instance, differences between in- and out-patient departments.

Studies have been conducted to evaluate the fabrication process of hand orthoses. A process model was built by Paterson et al. [17] to summarize activities of therapists during a traditional hand orthosis designing and making. The process was considered as a linear workflow with several iterations. The study by Stefanovich et al. [18] developed an evaluation process using the Delphi method and tested several tools to assess fabrication skills of therapy students. A survey was conducted by Ghoseiri et al. [19] to evaluate patients' satisfaction related to customization and using of their orthoses and prostheses in the clinic. Hagemann et al. [20] compared fabrication performances between two groups of hand therapy students who were trained to customize on human and model hands respectively. From the current state-of-the-art, only few researchers have quantitatively investigated real-time fabrication processes of experienced therapists in a clinical environment [21], [22]. The lack of quantitative data is a barrier to move towards a more effective data-driven approach to customize the fabrication process. Thus, a quantitative task analysis approach is explored further here.

Task analyses have been applied in literatures. Richardson et al. [23] assessed the complexity of assembly using Hierarchical Task Analysis with task variables derived from pre-designed instructions and studied the correlation between identified task variables and subjective ratings of difficulty from psychology undergraduates. Although, a statistical significance was found, the participants subjectively evaluated the difficulty through studying the booklet without actually performing assemblies. Thus, little information can be inferred, regarding real-time performances using this method. Wolever et al. [24] identified critical job tasks of health and wellness coaches by organizing a panel discussion of 15 experts. The importance and frequency of tasks were rated in a survey by a large group of professionals. Although professionals were involved in this study, tasks identified in the panel discussion were broad and detailed behaviors during tasks were not taken into consideration. In studies [25], [26], [27], Verbal Protocol Analysis (VPA) was applied to evaluate real-time behaviors of car drivers, train drivers and designers respectively through capturing information from their cognitive processes during tasks. Considering the diverse and implicit process of the orthosis fabrication, VPA was selected as an appropriate research method to collect and analyze data of the fabrication process, as rich data can be acquired from a naturalistic setting with detailed information of real-time behaviors of professionals. Time spent and frequency of tasks can be easily extracted from verbal data to quantitatively conduct tasks analysis [28]. The main purpose of applying VPA in this paper is to (1) quantitatively disclose the customization process and reveal detailed actions and intentions of therapists, and (2) understand how data from patients and therapists impacts on tasks of customization. This study is a step towards a data-driven approach to customize orthoses for improving fit through observing therapists in two clinical settings. Working models were elicited to identify data that is relevant to fit improvement. 
TABLE I: Background data of therapists and patients

\begin{tabular}{|c|c|c|c|c|}
\hline Therapists & In- or out-patient & $\begin{array}{l}\text { Years of } \\
\text { experience }\end{array}$ & $\begin{array}{l}\text { Number of } \\
\text { patients }\end{array}$ & $\begin{array}{l}\text { Average age of patients } \\
\text { with standard deviation }\end{array}$ \\
\hline Ao16 & Out & 16 & 4 & \multirow{2}{*}{$42.3 \pm 18.3$} \\
\hline Bo8 & Out & 8 & 4 & \multirow{2}{*}{$42.6 \pm 17.9$} \\
\hline Ci11 & In & 11 & 3 & \multirow{2}{*}{} \\
\hline Di7 & In & 7 & 7 & \\
\hline
\end{tabular}

\section{METHODS}

A 7-step method (see Fig. 1) is proposed here to generate working models of therapists: (1) collecting patients' conditions, i.e. number of joints to be stabilized; (2) collecting background information of therapists; (3) collecting verbal data during customization process; (4) processing verbal data; (5) calculating time consumption and iterations of tasks; (6) statistical analysis of data from step (1), (2) and (5); (7) Generating working models.

\section{A. Study set}

Data was collected from observing fabrication processes of 18 patients with hand injuries, with the average age of 42.4 (standard deviation $=17.5$ ), including eight out-patients and ten in-patients. Observations were conducted with four professional therapists with 16, 8, 11 and 7 years of working experience. They are referred to as Ao16, Bo8, Ci11 and Di7 in this paper, where 'o' or ' $\mathrm{i}$ ' respectively referring to the out- or in-patient department and the number indicating the years of experience. Each therapist was responsible for a number of patients, as shown in TABLE I. 18 adult patients who were prescribed with customized hand and wrist (h\&w) orthoses were selected by therapists and recruited in the study. All patients provided consent of participation independently and voluntarily, and they could withdraw from the study at any time without affecting their medical treatments. Several exclusion criteria were pre-defined to follow the ethical protocol closely and to eliminate extreme cases that were distinguished from general orthosis customization. Patients with the following conditions were excluded: (1) diabetes, hand paresthesia or sensation loss on hands; (2) mental illness or cognitive symptoms; and (3) patients in whole day care who could not provide independent consent to participant in the study. The criteria were also employed in our previous study [29]. All data collected were stored anonymously and confidentially, and the identification of all participants, including patients and therapists, could not be recognized. This study has been approved by the Xuzhou Central Hospital Biomedical Research Ethics Committee (XZXYLJ-20190222-003, 22/02/2019) and the Imperial College Research Ethics Committee (19IC5128, 26/04/2019).

\section{B. Data Collection}

Therapists were asked to think-aloud during orthosis fabrication to enable the rationale of activities to be better understood. A camera (Canon EOS 550D, Canon Inc.) was used to film the full customization process. To protect identities of patients and therapists, only hands were tracked and filmed. The therapist was asked to talk aloud their thoughts regarding the fabrication in real time and their voice was recorded using the camera. Since the data collection was set in a public hospital with a real live task, rather than an artificial task in a lab setting, interruptions from the environment were inevitable. When the therapist carried on the task as the interruption absented, the researcher reminded the therapist of the last verbal content to continue recording, and all interruptions were excluded from the data.

Some studies [30], [31] showed that the Think-aloud procedure would take more time for participants to complete tasks, since they require extra time to verbalize thinking. This may result in inaccuracy of time calculation of major phases and activities during fabrication. However, this study focuses on correlations and differences across major fabrication phases, thus extra time required for Think-aloud was considered evenly spent throughout the process. Another limitation of think-aloud method is the incompleteness of data due to three reasons: First, some therapists did not talk consistently as they were not used to talk aloud their thinking while performing tasks or they felt uncomfortable to say aloud in front of patients. Therapists were asked to practice several times performing the think-aloud technique on real patients, prior to the observation, in order to mitigate the effect of Think-aloud on their usual fabrication practice [32]; Second, some cognitive processes cannot be verbalized as some movements were conducted unconsciously [33], since the experienced therapists were familiar with the tasks; and third, if multiple tasks were performed by the therapist in a short time, it may be too intensive to verbalize all thoughts. Therefore, if the lack of verbalization was observed under the above circumstances, the researcher interrupted the fabrication process and asked the therapist for explanation of the new movements. In all observations, therapists paused their processes and explained. Since they are experienced therapists, short time of interruption did not affect the fabrication process.

\section{Data Processing}

Verbalized data was processed through five steps: 1) transcription, 2) segmentation, 3) coding, 4) validation and, 5) calculating time consumption and the number of iterations. Verbalized data from recorded videos was transcribed into written text manually. 


\begin{tabular}{|c|c|c|}
\hline 1st level code & 2nd level code & 3rd level code \\
\hline \multicolumn{3}{|c|}{ Main Phases and Subordinate Activities of Fabrication } \\
\hline \multirow{11}{*}{ Preparation } & \multirow{2}{*}{ Condition evaluation } & Check diagnosis report \\
\hline & & Check X-ray image \\
\hline & \multirow{2}{*}{ Orthosis design } & Decide stabilised joints \\
\hline & & Decide released joints \\
\hline & \multirow{4}{*}{$\begin{array}{l}\text { Pre-fabrication } \\
\text { organisation }\end{array}$} & Organise gauzes on the hand \\
\hline & & Organise tools for fabrication \\
\hline & & Position the hand \\
\hline & & Cover hand with cling film or fabric \\
\hline & \multirow{3}{*}{ LTT preparation } & Measure dimensions \\
\hline & & Cut LTT based on dimensions \\
\hline & & Soften LTT \\
\hline \multirow{8}{*}{ Shape forming } & \multirow{5}{*}{$\begin{array}{l}\text { Generate orthosis } \\
\text { form }\end{array}$} & Apply LTT on the hand \\
\hline & & Cut and fold LTT \\
\hline & & Add enhancement structure \\
\hline & & Mould LTT over the hand \\
\hline & & Facilitate LTT hardening \\
\hline & \multirow{2}{*}{$\begin{array}{l}\text { Remove extra } \\
\text { material }\end{array}$} & Draw cutting references \\
\hline & & Cut LTT according to references \\
\hline & \multicolumn{2}{|l|}{ Re-softening } \\
\hline \multirow{7}{*}{ Adjustment } & \multirow{3}{*}{ Edge treatment } & Heat and smoothen edges \\
\hline & & Bend or roll edges \\
\hline & & Cover edges with foam strips \\
\hline & \multirow{3}{*}{ Comfortable fitting } & Attach paddings on the hand \\
\hline & & Stick paddings on the orthosis \\
\hline & & Release pressure under the orthosis \\
\hline & \multicolumn{2}{|c|}{ Tries on patients during adjustment } \\
\hline \multirow{4}{*}{ Fastening } & \multirow{3}{*}{ Fasten Velcro } & Cut Velcro \\
\hline & & Attach Velcro \\
\hline & & Secure Velcro \\
\hline & \multicolumn{2}{|c|}{ Tries on patients during fastening } \\
\hline \multicolumn{3}{|c|}{ Interactions During Fabrication } \\
\hline \multirow{4}{*}{ Communicating } & \multicolumn{2}{|c|}{ Ask for patients' compliance } \\
\hline & \multicolumn{2}{|c|}{ Ask for patients' feedback } \\
\hline & \multicolumn{2}{|c|}{ Educate patients } \\
\hline & \multicolumn{2}{|c|}{ Answer patients' questions } \\
\hline \multirow{4}{*}{ Observing } & \multicolumn{2}{|c|}{ Check fitting and tightness } \\
\hline & \multicolumn{2}{|c|}{ Check mobility of released joints } \\
\hline & \multicolumn{2}{|c|}{ Check stabilised joints } \\
\hline & Check skin conditions & \\
\hline Explaining & & \\
\hline
\end{tabular}

Fig. 2: The coding scheme. The scheme was also used in our previous study [29].

Fig. 2 Alt Text: A three columns list of codes including seven 1st level codes, nine 2nd level codes and 38 3rd level codes as demonstrated in details in Section II-C.

Irrelevant data to therapists' thoughts of the customization, including communications between the therapist and other hospital staff, patients and family members were not considered as eligible data and were eliminated during transcription. Voice and movements of therapists were both transcribed. For example, when the therapist said: "This joint of the thumb will be stabilized", the therapist was pointing at the thumb carpometacarpal (CMC) joint. Thus, referred to the movement observed from the video, the transcription outcome was "This CMC joint of the thumb will be stabilized", to avoid confusion. The communication between the therapist and the patient was also transcribed. Furthermore, if no verbalized data was gained during a movement of the therapist, simply because the therapist forgot or was too busy to speak, the movement was described in the text. These descriptions were clarified and confirmed by the therapist during the transcription.

Segmentation was undertaken to divide the transcribed content into smaller sentences or paragraphs. Chi [34] proposed several methods to segment verbal data based on size, characteristics, correspondence and necessity of segmentation. In this case, segmenting according to the two criteria [29] were found efficient to generate codable utterances. First, continuous sentences with content of similar tasks or movements which were conducted with the same intent, were segmented into the same category. Second, a single sentence could be categorized into multiple segments, if several movements or tasks with different intents were observed. For instance, the sentence "I place his hand in the functional position and apply the softened material on it" was divided into two parts. First, "I place his hand in the functional position" with the task of positioning the hand, and second, "apply the softened material on it" with a different task of using the material.

The coding scheme (Fig. 2) was developed using a bottom-up approach with three levels of codes as it helps constructing codes by interpreting segmented transcriptions [35] which was more suitable for this study. Feedback from the four therapists 

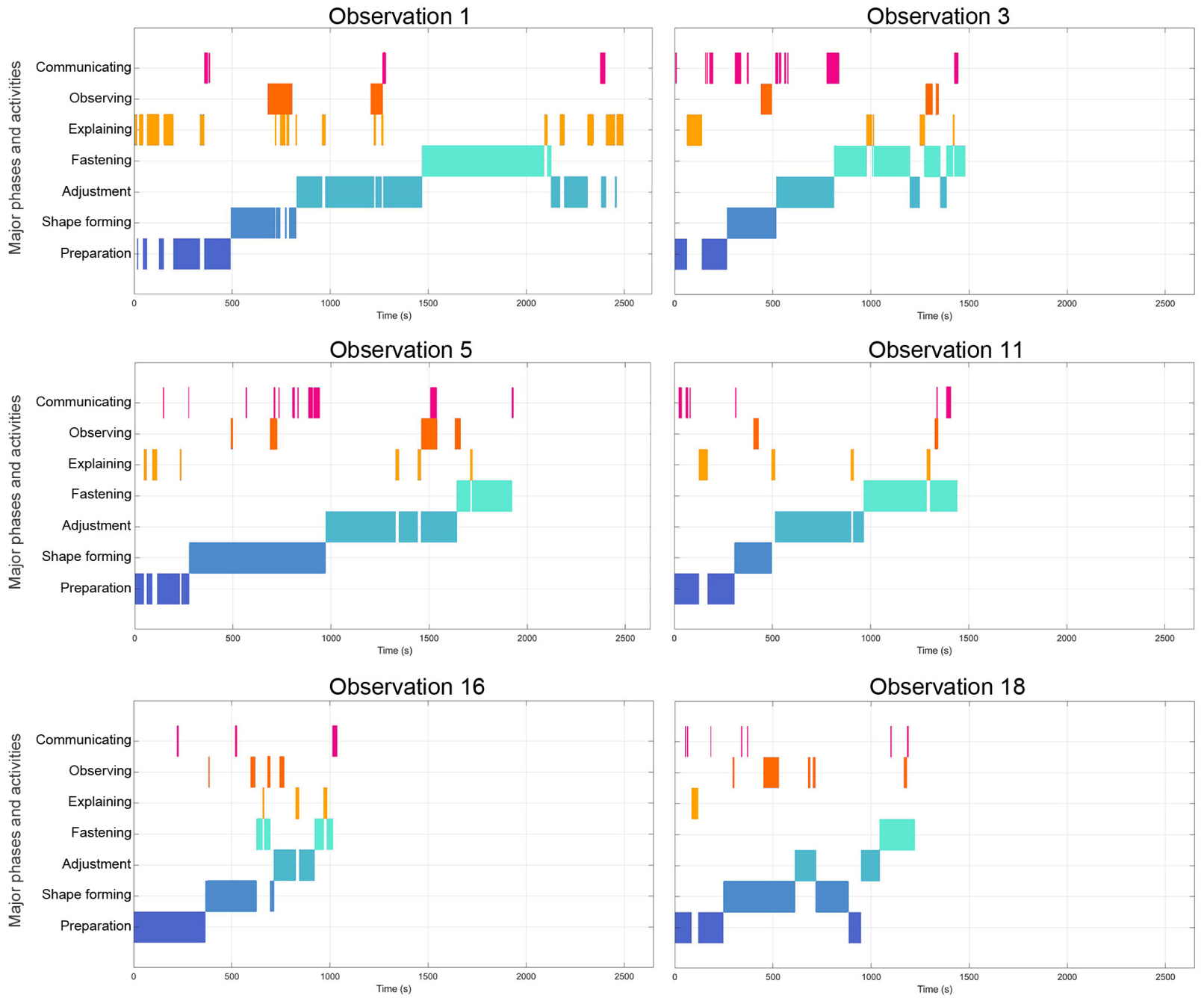

Fig. 3: Examples of observations regarding time spent on major phases and activities of fabrication

Fig. 3 Alt Text: Six time charts of six observations with time intervals of seven major phases and activities, i.e. the seven 1st level codes.

was sought to inform the coding scheme design. There are seven first level codes representing major phases and activities during customization processes. For example, the code "shape forming" stands for the major phase of customization that the therapist applied the LTT material (a low temperature thermoplastic material for splinting) on the patient's hand and mold the shape of the orthosis. Some first level codes were created by categorizing several subordinate tasks which were defined by nine second level codes. Moreover, first or second level codes were generated by merging the 38 third level codes which were initially derived from each segment, demonstrating individual movements or tasks. For instance, the code "draw cutting references" was generated based on the segment "I am using a pen to draw a circumferential line on the LTT material around the patient's thumb interphalangeal joint to define an area to be cut." As the bottom-up approach was adopted, new third level codes were revealed and added to the coding scheme when reviewing new transcriptions. Additionally, second level codes were not necessary between some first and third levels as the first level codes can be directly abstracted from the third level codes, such as "communicating" and "observing". They consist of several independent movements which cannot be classified into second level codes. The code "explaining" is considered as an independent first level code as the therapists had a single intention of explaining to the researcher rather as part of their tasks.

The Cohen's Kappa was conducted by two researchers to confirm the inter-coder reliability of the coding scheme. Several segmented transcriptions and the coding scheme were provided to the second researcher who was asked to assign the code(s) to corresponding segments. The result of Cohen's Kappa (0.83) shows good agreement between the two researchers. Another Kappa test was conducted to evaluate if the code occurred or completed at the same time with time tolerance set as 1 second according to [28]. The value of Kappa between two researchers is 0.73 showing substantial agreement.

Six examples of time charts regarding how time was organized during major phases (i.e. preparation, shape forming, adjustment and fastening) and tasks (i.e. communicating, observing and explaining) are shown in Fig. 3. The time spent 
a

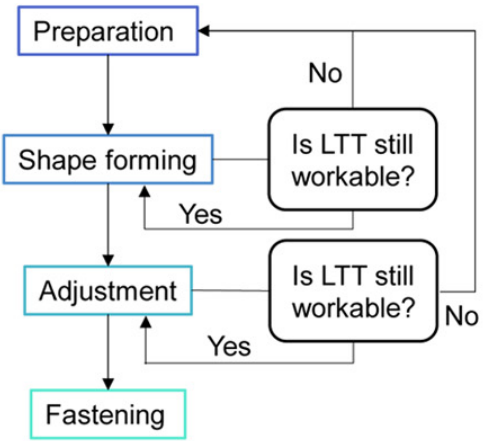

b

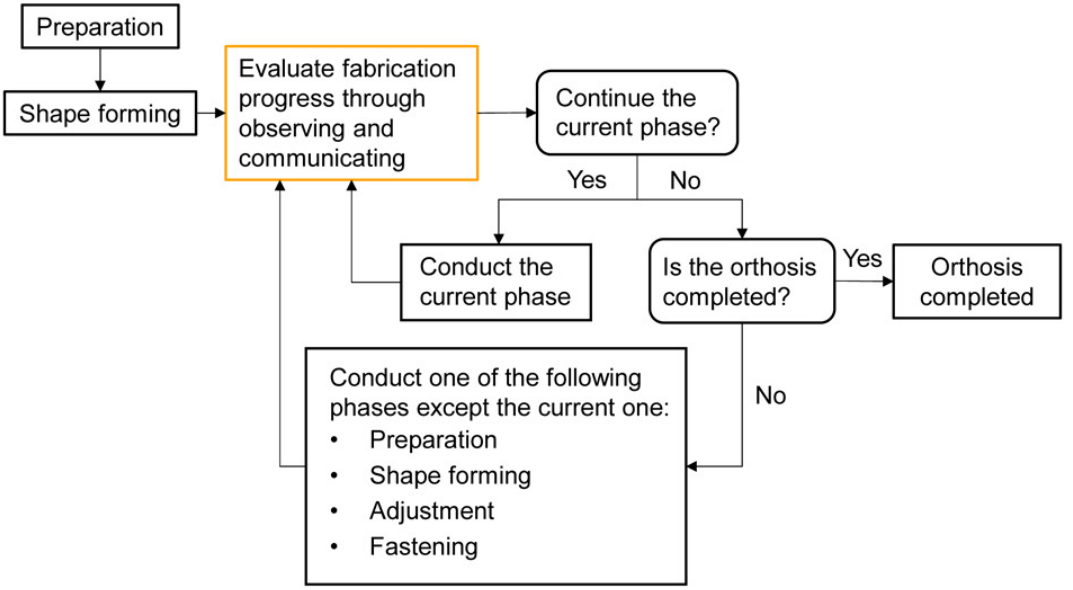

Fig. 4: Comparison between (a) the linear fabrication process and (b) the non-linear fabrication process (it always starts with preparation followed by shape forming).

Fig. 4 Alt Text: Two workflow diagrams: (a) A single workflow starting with "Preparation" and followed by "Shape forming", "Adjustment" and "Fastening". The workflow may restart depending on whether the LTT material is workable at shape forming and adjustment phases. (b) A diagram starts from "Preparation" to "Shape forming" followed by a circular workflow. Any phase may occur after "Shape forming" depending on therapist's evaluation on the current progress of fabrication until it is considered completed.

on major phases or activities were determined by adding up the time consumption of movements or tasks represented by the third level codes. It can be noted that the time duration of "explaining" could be found throughout the fabrication while other activities stopped, as the therapist was explaining a task when the researcher interrupted the process under the circumstances demonstrated in section II-B. Moreover, the activities described by the two first level codes "observing" and "communicating" took place at the same time while other movements were conducted during the four major phases. Additionally, the number of iterations of conducting tasks defined by the third level codes, including "re-softening", "tries on patients during adjustment" and "tries on patients during fastening", was counted.

After all the data was coded, data regarding time spent and iterations of tasks was analyzed to evaluate their impact on the fabrication, including (1) number of stabilized joints, (2) fabrication time, (3) time spent on observing, (4) time spent on co-occurrence of observing and communicating, (5) times of re-softening and (6) times of trying on patients. The Shapiro-Wilk test was conducted and showed the data (2) has normal distribution ( $p>0.05)$, whereas (1), (5) and (6) were non-parametric data. The Mann-Whitney U test was conducted to investigate if data (1) and (5) were different between in- and out-patients. The one-way ANOVA was employed to test if fabrication time was different among therapists. Moreover, the relationship between re-softening times and time spent on shape forming was assessed using the Spearman's Rho test.

\section{RESULTS AND DISCUSSIONS}

\section{A. Order of fabrication phases}

Although the orthosis customization in literature is considered as a linear process [17], analysis from the 18 observations shows some non-linear processes in practice. These have been summarized in Fig. 4. From the linear fabrication process, the four major phases were operated in an order - preparation, shape forming, adjustment and fastening. However, abrupt changes of sequences were found in seven out of 18 observations. For instance, in observation 3 (see Fig. 3), phases of adjustment and fastening were conducted in a zigzag order, as the orthosis was adjusted twice during the fastening stage. In observation 16, shape forming is interrupted by fastening for 61 seconds. Specifically, the therapist fastened the Velcro (the task belongs to the fastening phase) on the semi-rigid LTT material which was applied to the participant's hand during the shape forming phase. As explained by the therapist, since the shape of the orthosis was successfully molded, attaching Velcro would not affect the current progress. To save time, fastening was conducted interrupting the shape forming phase. The non-linear process can be explained that the therapists were so familiar with the customization that major phases could be conducted in a flexible order to achieve an efficient fabrication. However, the non-linear process may hinder data collection during targeted phases to improve fit, as when these tasks will be conducted is non-standard and unpredictable. Therefore, it is essential to understand the time spent and frequency of the main activities of therapists to identify where useful data can be collected.

\section{B. Complexity of fabrication}

According to the feedback of the four therapists, the complexity of a fabrication process is difficult to be quantified, and is mainly affected by three factors, including patient's condition, patient compliance and types of orthoses. Since all patient 


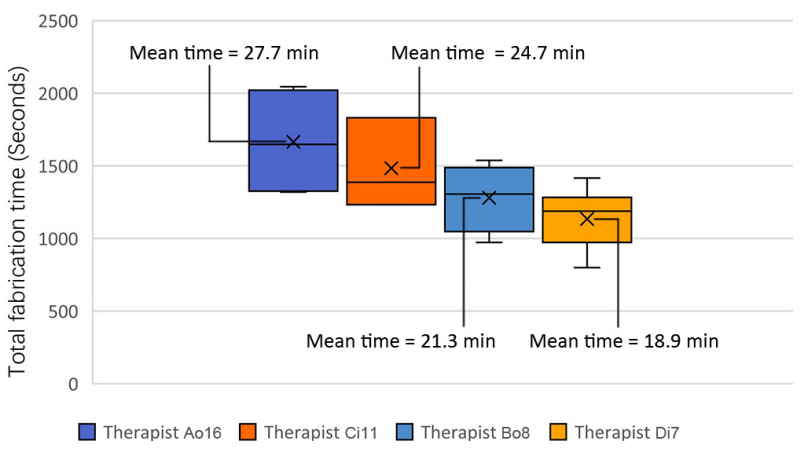

Fig. 5: Total fabrication time of each therapist [29].

Fig. 5 Alt Text: A box plot of total fabrication time of the four therapists with average value marked as $27.7 \mathrm{~min}, 24.7 \mathrm{~min}$, $21.3 \mathrm{~min}$ and $18.9 \mathrm{~min}$ for therapists Ao16, Ci11, Bo8 and Di7 respectively.

participants showed high compliance during fabrications and the static h\&w orthosis was the only type employed, the conditions of participants were considered as the main contributor affecting the complexity of fabrication. Although the patient conditions were varied and could not be easily categorized, the number of stabilized joints could be used as an indicator of complexity.

As a h\&w orthosis aims to stabilize affected joints and retain flexibility of unaffected joints [15], the more injured segments of a hand that require more functional parts of an orthosis, leading to a more complex fabrication process [36]. For instance, three joints were stabilized of an out-patient participant with a single fracture at the fifth metacarpal bone, whereas for another in-patient participant with multiple injured area, 13 joints were stabilized in various angles with a more complicated fabrication. Additionally, according to the principle of orthosis stabilization [15], the primarily affected joint and the nearest joints on the same kinetic chain should be immobilized. Therefore, the number of stabilized joints is directly related to the condition and reflects the complexity of fabrication.

A Mann-Whitney $U$ test was conducted to investigate if there was a difference in the number of stabilized joints between in- and out-patients. The result showed that in-patients had more stabilized joints than out-patients $(\mathrm{p}<0.05)$ with the mean number of 3.5, 2.5, 5.3 and 7.4 for therapists Ao16, Bo8, Ci11 and Di7 respectively, which indicated a more complex fabrication process at the in-patient department. This finding indicated that data from in- and out-patient should be compared and evaluated separately.

\section{Total fabrication time and time spent on major phases}

A one-way ANOVA was performed to clarify if the total fabrication time was different among the four therapists and a significant difference was revealed $(\mathrm{p}<0.05)$. The box plots of total time consumption by therapists with all corresponding patients are reported in Fig. 5. It can be noted that more fabrication time was spent by therapists with more working experience. Though this trend may be abstract as the number of therapists observed was small, it may be explained by two reasons. First, more time may be spent by more experienced therapists on interacting with patients in details, e.g. checking the skin condition and asking feedback of patients regarding comfort. However, less experienced therapists may not pay adequate attention on these details. Second, the assessment and modification of completed orthoses may cost more time for more experienced therapists than less experienced therapists. Similar results were gained in the study [37] showing more experienced physical therapists spent more time on seeking information from patient and evaluating treatment. Therefore, less experienced therapists may neglect some information that was obtained by more experienced therapists, which are shown intuitively in working models in section III-G.

The Fig. 6 shows the average percentage of time consumption on the four major phases performed by the four therapists. It can be noted that therapists Ao16 and Bo8 (out-patient therapists) separated similar proportions of fabrication time into the four major phases. Also, the identical arrangement of time can be seen between therapists Ci11 and Di7 (in-patient therapists). The lowest time proportions of $15.45 \%$ and $13.55 \%$ were spent on the shape forming phase by therapists Ao16 and Bo8 respectively. By contrast, in-patient therapists spent the largest proportion of time during shape forming with $36.60 \%$ by therapist Ci11 and $31.83 \%$ by therapist Di7. Moreover, the fastening phase also reveals the different strategy of time arrangement between inand out-patient therapists. Specifically, therapists Ao16 and Bo8 devoted the most proportion of time to build fasteners with $39.33 \%$ and $39.86 \%$ respectively. However, the fastening phase took the smallest proportion of fabrication time (14.99\%) by in-patient therapists.

These findings indicated that out-patient therapists paid particular attention to the fastening phase. High durability of orthosis can be achieved with more careful fastening, thus minimizing the risk of losing tightness after the patient has left the hospital, and also reducing additional appointments of patients for orthosis adjustment and repair. In-patient therapists spent more time on shaping forms of orthoses. This may be due to two reasons: (1) Post-surgery in-patients required more care for open wounds during shape forming. For example, regular check is essential to ensure that orthoses do not place excessive pressure on the 


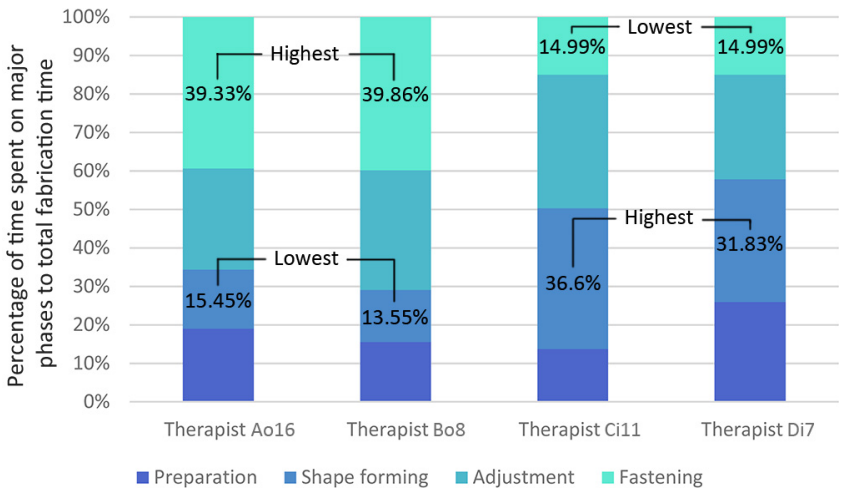

Fig. 6: Average percentage of time spent on major phases [29].

Fig. 6 Alt Text: A segmented bar chart with four bars for all therapists. Each bar is divided into four segments corresponding to the four major phases with the highest and the lowest percentage marked at each bar.

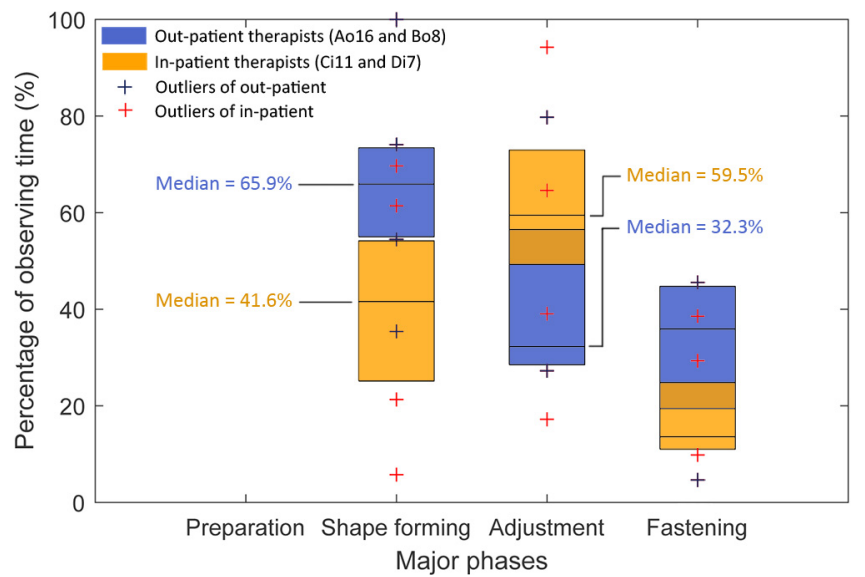

Fig. 7: Percentage of the total observing time spent on each major phase.

Fig. 7 Alt Text: A box plot of percentage of total observing time spent on the four major phases. Data from in- and out-patient therapists were separated. The median values of percentages were reported with $65.9 \%$ and $41.6 \%$ of total observing time spent on shape forming for out- and in-patient therapists respectively, and 59.5\% and $32.3 \%$ spent on adjustment by in- and out-patient therapists respectively.

neurovascular bundle near wounds of post-surgery patients with Dupuytren's contracture [38]. (2) The orthosis shape molded on the hand of an in-patient was more complicated, since more hand joints were immobilized than out-patients as clarified in section III-B. Additionally, the durability of orthoses may not be the priority for in-patients as they live in the hospital with easy access to repair by therapists. Similar results have also been revealed in the study [29].

Although therapists applied similar techniques and materials throughout the process, in- and out-patient therapists showed differences and by analyzing the time spend enabled these differences to be visible. For instance, to improve fit for in-patients, there is higher chance that valuable data can be collected from the shaping forming tasks, such as the geometrical data of the orthosis and the locations of enhancement structures. On the other hand, to develop methods to improve fit for out-patients, data form the fastening phase may provide critical information, such as the distribution and the tightness of fasteners. Also, the time spent on shape forming and fastening can be used to evaluate whether the fit improving method facilitates tasks during the two phases.

\section{Observing and communicating}

The two first level codes, "observing" and "communicating", describe interactions between therapists and patients through which the therapists gather information from patients to guide further fabrication. Therefore, the code "observing" as well as the co-occurring of "observing" and "communicating" were analyzed to identify the phases in which these activities took place and the difference between in- and out-patient therapists.

1) Observing: The percentage of total observing time spent on the four major phases between in and out-patient therapists is reported in Fig. 7. It can be noted that observing did not occur during the preparation phase and mainly occurred during shape forming and adjustment phases. Thus, compared with preparation and fastening phases, the shape forming and the adjustment 


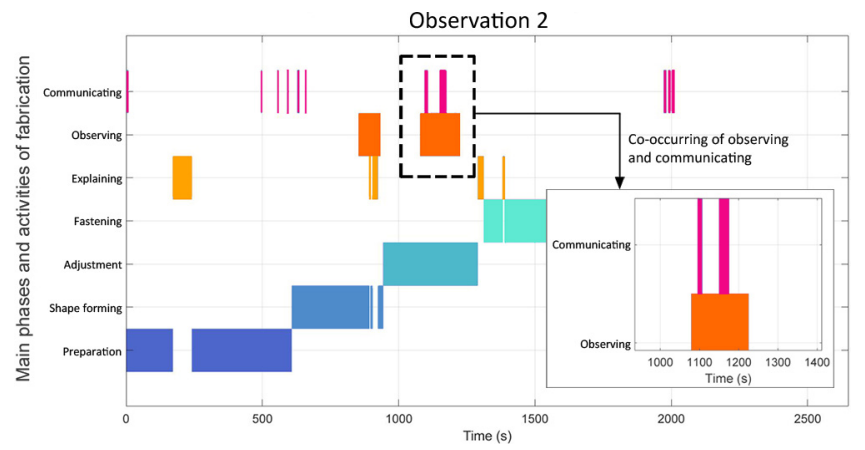

Fig. 8: An example of co-occurrence of observing and communicating (Observation 2). When the therapist was checking the tightness of the orthosis (observing), the therapist asked the patient twice (at $1095 \mathrm{~s}$ and $1150 \mathrm{~s}$ ) regarding the sensation of contact pressure at two locations on the hand (communicating)

Fig. 8 Alt Text: A time chart of major phases and activities conducted during the No.2 observation. A time interval is zoomed in showing two co-occurrences of observing and communicating.

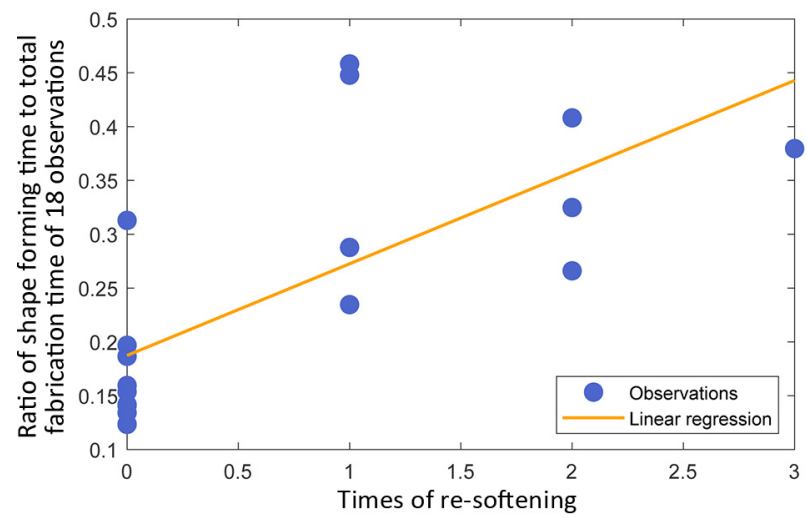

Fig. 9: Correlation between the re-softening times and the time spent on the shape forming phase over 18 observations.

Fig. 9 Alt Text: A scatter plot showing the ratio of shape forming time to total fabrication time of all subjects with their corresponding times of re-softening. The line of linear regression is also plotted.

phases are where the most information is gained through observing. For out-patient therapists, they spent the majority of observing time at the shape forming phase, with the mean of 57.6\% for therapist Ao16 and 73.4\% for therapist Bo8 across their patients, both more than the in-patient therapists. For therapists Ci11 and Di7, they both spent the highest percentage of time observing at the adjustment phase with means of $60.9 \%$ and $48.3 \%$ respectively, both more than out-patient therapists.

This result identifies two phases when information was gathered through observing to assess fabrication. As observing was conducted mainly for evaluating fabrication progresses, this result indicates that the assessment of whether the orthosis has achieved the correct fit mostly occurred at the shape forming phase for out-patient therapists, but at the adjustment phase for in-patient therapists. This difference may be explained as the emphasis of work for out-patient therapists is to customize a general shape of orthoses at the shape forming phase to fit to single or few affected joints, which is a rough fabrication process, but adjusting the general shape to fit in more complex conditions of patients (fine fabrication) plays a more important role for in-patient therapists.

Therefore, through identifying the specific phases when therapists assessed fitting through observing, the main data to evaluate fit, i.e. what therapists were observing, can be easily identified, and collected in large scale during the targeted phase. For instance, angles of released joints can be gathered during shape forming for out-patients, and data related to edge treatment can be collected during adjustment for in-patients.

2) Co-occurrence of observing and communicating: Though observing and communicating are independent activities scattered throughout the customization process, nine observations show that observing and communicating took place simultaneously, with six observations showing them co-occurring at the adjustment phase (e.g. Fig. 8), two observations at the fastening phase and one observation at both shape forming and adjustment phases. To identify which therapist spent more time conducting the two behaviors at the same time, the mean of ratios between the total co-occurring time and the total fabrication time of their corresponding observations was calculated. The analysis found the highest ratio from therapist Ci11 (1.42\%) followed by therapist Di7 (1.0\%), therapist Ao16 (0.56\%) and therapist Bo8 (0.33\%). Both in-patient therapists (Ci11 and Di7) have higher ratios than out-patient therapists. Additionally, the co-occurring time was found in six of ten (60\%) in-patient observations, but 

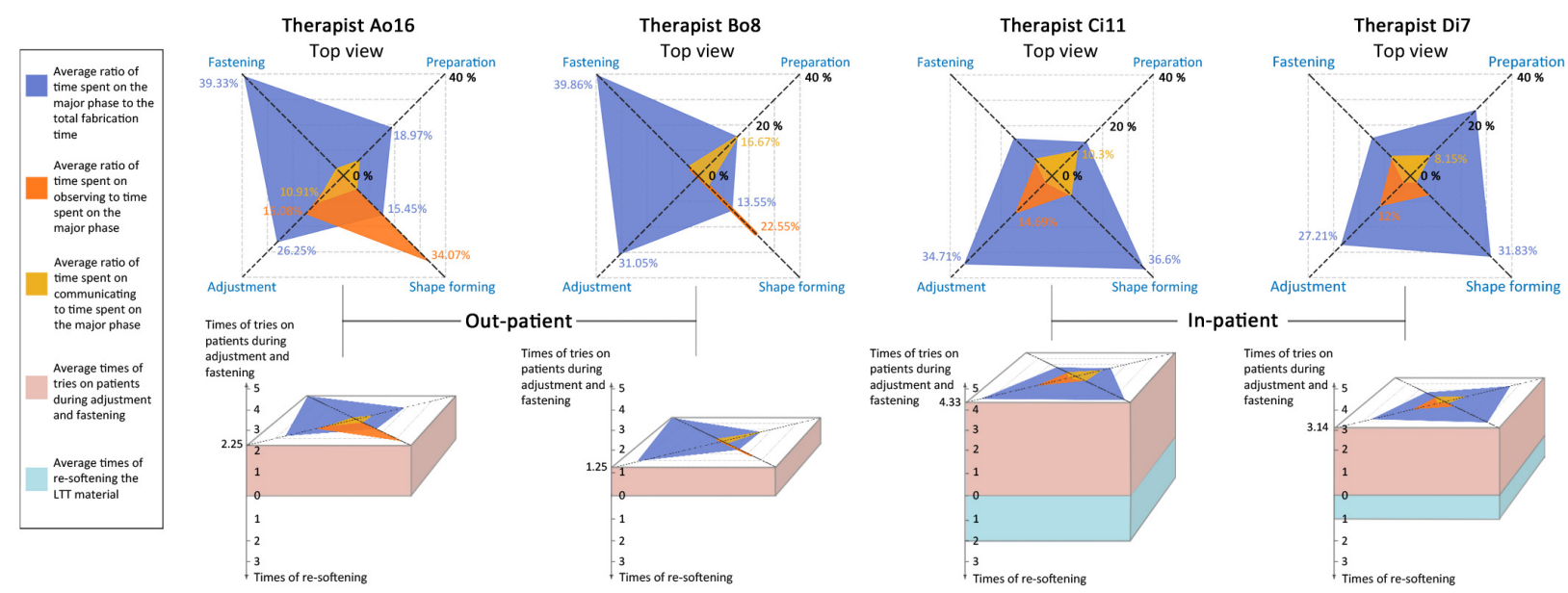

Fig. 10: The 3D model and its top view represent the working model of individual therapist. The mean of ratios of time spent on each major phase to total fabrication time (blue), the mean of ratios of time spent on observing during each major phase to time spent on each major phase (orange) and the mean of ratios of time spent on communicating during each major phase to time spent on each major phase (yellow) are shown. The upward and downward numbers of the vertical axis show the times of conducting "tries on patients" and "re-softening".

Fig. 10 Alt Text: Each 3D working model is a cuboid. Its top view is a square radar chart with the four major phases at each corner. The cuboid is separated into two parts. The height of the top part indicates average times of "tries on patients" and the height of the bottom part shows the average times of "re-softening".

$38 \%$ of out-patient observations, indicating observing and communicating were more frequently applied together in in-patient fabrications.

Hence, these results revealed that the co-occurrence mostly took place during adjustment and occurred in in-patient more than out-patient. As this interaction between therapists and patients was conducted to obtain more information to guide fabrication, for example, the therapist observed the fitting and asked the patient's feedback regarding tightness, this finding discloses where uncertainty predominantly occurred and informs the focus of further research to understand the reasons for the interaction and the information that is gained to overcome uncertainty.

\section{E. Re-softening}

The code "re-softening" refers to the procedure when the semi-finished orthosis is placed back in the water bath to re-soften the material during the shape forming phase. It takes place as the material has become rigid before shape forming is completed or the orthosis has been shaped incorrectly. It was only conducted by in-patient therapists. Hypothetically, times of re-softening is correlated to the shape forming time, as more times of re-softening lead to longer molding time required to form the orthosis correctly. The linear regression of the re-softening times and the ratios of the shape forming time to the total fabrication time is shown in Fig. 9, suggesting a monotonically increasing relationship between the two variables (Spearman's Rho correlation coefficient $=0.76, \mathrm{p}<0.01$ ).

Thus, except time spent on shape forming, the re-softening times also help to assess the efficiency of the phase. Although outpatient therapists did not re-soften materials, it does not mean in-patent therapists have lower skills, as they are all experienced therapists. But the complexity of in-patient fabrication hindered the phase to be completed shortly. This finding indicated that data regarding the reasons of the re-softening should be targeted in further studies to reduce the number of iterations.

\section{F. Tries on patients during adjustment and fastening}

The two third level codes, "tries on patients during adjustment" and "tries on patients during fastening" were analyzed together and indicate therapists trying the semi-finished orthosis on the patient's hand to evaluate the fitting. A Mann-Whitney test was conducted to assess independence of trying times between in and out-patient observations. The result shows that the number of trying times is significantly higher for in-patient therapists than for out-patient therapists $(\mathrm{p}<0.01)$. It can also be noted that, in the same department (in- or out-patient), more experienced therapists tend to conduct a higher number of tries on patients than less experienced therapists, with the mean of trying times 4.33 for therapist Ci11 higher than therapist Di7 (mean $=3.14)$, and therapist Ao16 $($ mean $=2.25)$ is also higher than therapist Bo8 (mean $=1.25)$.

Overall, more tries were conducted by in-patient therapists as they tackled more complex conditions which required repetitive evaluations. The more experienced therapists tried more times on patients, hence spending more time on fabrication than the less experienced therapist, as explained in section III-C. Similarly, with the data of re-softening that can be used to evaluate 


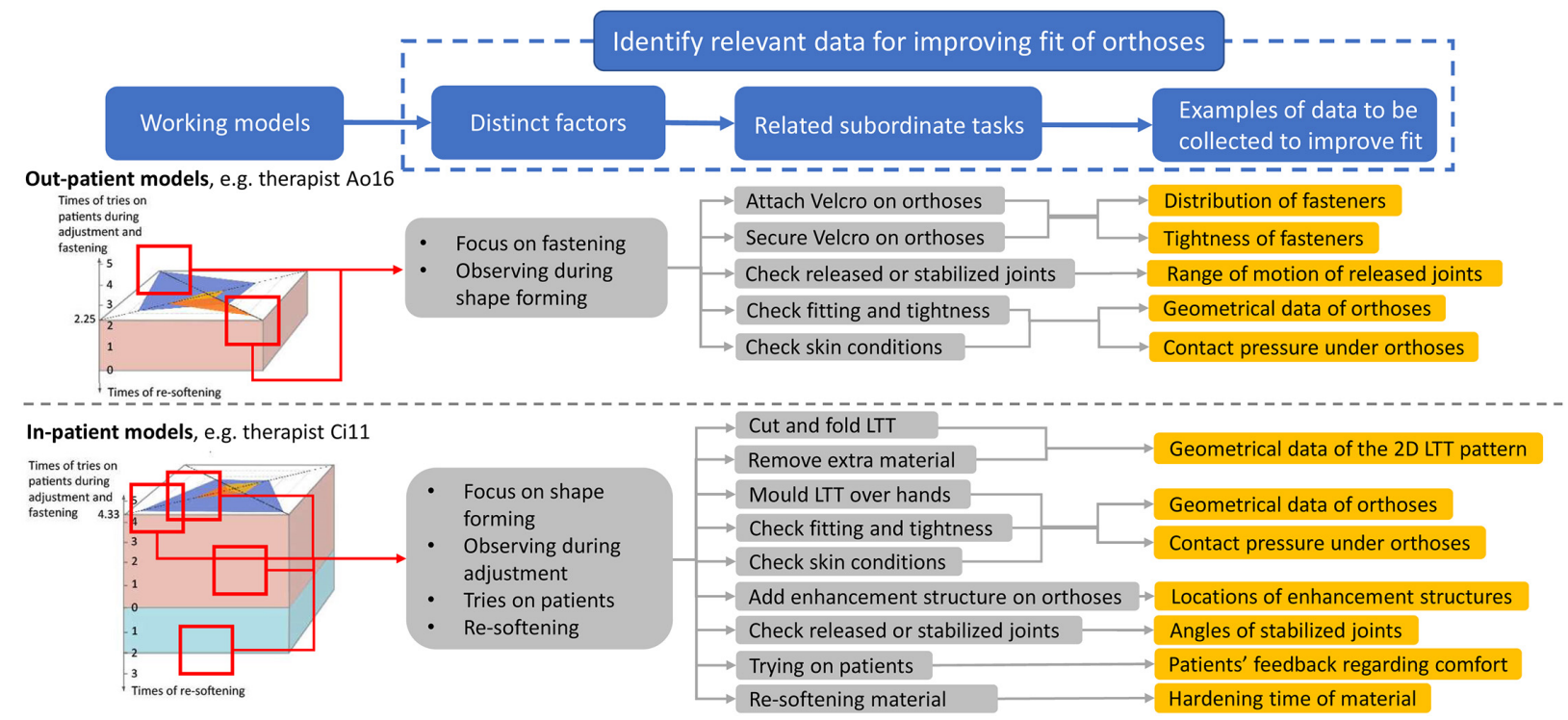

Fig. 11: Distinct factors identified from the working models that involve specific subordinate tasks inform data to be collected on a large scale in further studies. Though the working model does not directly deliver methods to improve fit of orthoses, it provides a clear direction to reveal data with high impact on improving fit of orthoses depending on different clinical contexts. Fig. 11 Alt Text: Two four-section diagrams demonstrate how to extract data to be collected to improve fit from working models in further studies. Two working models of therapists A016 and Ci11 are used as examples in section 1. Distinct factors and related subordinate tasks are listed in section 2 and 3. Several examples of data to be collected are shown in section 4.

shape forming, the data of tries can help assess the efficiency of adjustment and fastening phases. By knowing this data, the difficulty of fitting can be estimated, and the data should be reduced if an effective method to facilitate fitting is applied.

\section{G. Working models of therapists}

Working models of therapists were derived from the findings across 18 patients observed in this study. A 3D model was created to illustrate the ratios of time spent on major phases, observing and communicating (Fig. 10). The number of times of conducting re-softening and tries on patients is also presented at the vertical axis of the model. An identical pattern can be seen between therapists Ao16 and Bo8 (out-patient), and therapists Ci11 and Di7 (in-patient), showing two distinct working patterns for in- and out-patient departments. Moreover, the height of the working model indicates the complexity of the fabrication, i.e. the taller the cuboid refers to a more complicated fabrication. The working models intuitively draw out therapists' performance of fabrication tasks, revealing their main intentions of work. For instance, comparing between therapist Ao16 and Bo8, and between therapist Ci11 and Di7, models of more experienced therapists occupy more orange area (i.e. spent more proportional time on communicating with patients), especially during the adjustment phase, and are taller in the pink direction (i.e. conducted more trials of orthoses on patients). Moreover, based on the working models, data with high impact on improving fit can be collected on a large scale from specific tasks as shown in Fig. 11 for further studies, for example, distribution of fasteners, angles of stabilized joints and hardening time of material etc.

\section{H. Limitations}

The proposed method has two main limitations. (1) Due to the data collection method, observations with the Thinking-aloud and the time needed to analyze the data, it requires a lot of effort to analyze data from large sample size. Thus, 18-observation is a relatively small sample size and findings although showing distinct patterns between in- and out-patient, cannot represent all customizations of h\&w orthoses. Also, since it was difficult to arrange for the same therapist to work in both in- and out-patient departments, it was possible that differences of working models between in- and out-patient therapists were caused by the personal working habit of the more experienced therapist which may be followed by less experienced therapists in the same department. However, as these therapists were all experienced in fabrication, the differences are likely due to the complexity of fabrication rather than a lack of knowledge from the therapist. Collaboration with multiple clinical centers would enhance the validity of the collected data and may reveal more working patterns. (2) The method cannot directly provide data for improving fit, but identifies the data needed and provides a focus of where high impact data can be found in the process, therefore reducing the time to gather data by alleviating the need to observe the entire process. For example, although the data of proportional time spent on observing during adjustment by in-patient therapists cannot be directly applied to improve fit, by knowing where most of this time is spent, information for evaluating fit (e.g. skin condition was observed at several 
locations on the hand) can be easily found during specific time (observing time during adjustment). Thus, methods to assess skin condition, e.g. contact pressure [13], can be developed in further studies.

\section{CONCLUSIONS}

This study proposed a method to generate working models of therapists regarding fabricating hand and wrist orthoses for patients with hand injuries in the clinical environment. A non-linear and unpredictable fabrication process was revealed and extended the traditional linear fabrication process model [17]. The long-term aim of this research is to develop a data-driven approach to improve fit of orthoses. This preliminary study was essential to: understand which factors and data influence the customization; disclose where information with high impact can be received from the fabrication process and reveal the need of different types of data for improving fit depending on different clinical contexts. Several key data were found with impact on the fabrication from the working models generated. The data of the number of stabilized joints defined the complexity of the fabrication and were validated by another critical data, i.e. the number of tries on patients, which influenced the total fabrication time. These two data can be used to evaluate the geometrical complication of an orthosis. Moreover, the time spent specifically on the shape forming and fastening phases were also identified as where key data can be collected, as well as the data regarding the proportional time spent on observing during the shape forming and adjustment phases. The two types of data revealed the phases that weigh most and may provide highly valuable information of improving fit. Additionally, the times of re-softening was identified as the key data only for in-patient fabrication, significantly affecting the efficiency of shape forming. All the identified data disclosed distinct working behaviors between in- and out-patient therapists. Overall, in-patient fabrication shows a greater degree of complexity than out-patient and whether the patient was in- or out-patient should be taken into prior consideration before collection of these data. The working model can be applied to other orthoses or prostheses to enable understanding of their fabrication processes, mitigating confusion caused by the complexity and educating hand therapy students.

\section{DECLARATION OF COMPETING INTEREST}

The authors declare that they have no known competing financial interests or personal relationships that could inappropriately influence the work reported in this paper.

\section{ACKNOWLEDGMENT}

This study was conducted at the Imperial College London and the Xuzhou Central Hospital. The project was partially supported by the Affiliated Xuzhou Rehabilitation Hospital of Xuzhou Medical University, the Xuzhou Central Hospital, the China Scholarship Council and the Imperial College London. The authors would like to thank all participants who have contributed to this research.

\section{REFERENCES}

[1] N. England, "Improving the quality of orthotics services in england," 2015.

[2] F. Sandford, N. Barlow, and J. Lewis, "A study to examine patient adherence to wearing 24-hour forearm thermoplastic splints after tendon repairs," Journal of Hand Therapy, vol. 21, no. 1, pp. 44-53, 2008.

[3] A. Lucas-Cuevas, P. Perez-Soriano, J. Priego-Quesada, and S. Llana-Belloch, "Influence of foot orthosis customisation on perceived comfort during running," Ergonomics, vol. 57, no. 10, pp. 1590-1596, 2014.

[4] J. Langley, S. Pancani, K. Kilner, H. Reed, A. Stanton, N. Heron, S. Judge, A. McCarthy, S. Baxter, C. Mazz'a et al., "A comfort assessment of existing cervical orthoses," Ergonomics, vol. 61, no. 2, pp. 329-338, 2018.

[5] E. Taylor, J. Hanna, and H. Belcher, "Splinting of the hand and wrist," Current Orthopaedics, vol. 17, no. 6, pp. 465-474, 2003.

[6] J. Buurke, J. Grady, J. De Vries, and C. T. Baten, "Usability of thenar eminence orthoses: report of a comparative study," Clinical rehabilitation, vol. 13, no. 4, pp. 288-294, 1999.

[7] J. S. Murray, C. Noonan, S. Quigley, and M. A. Curley, "Medical device-related hospital-acquired pressure ulcers in children: an integrative review," Journal of Pediatric Nursing, vol. 28, no. 6, pp. 585-595, 2013.

[8] T. Richards, R. Clement, I. Russell, and D. Newington, "Acute hand injury splinting-the good, the bad and the ugly," The Annals of The Royal College of Surgeons of England, vol. 100, no. 2, pp. 92-96, 2018.

[9] P. McKee and A. Rivard, "Orthoses as enablers of occupation: client-centred splinting for better outcomes," Canadian Journal of Occupational Therapy, vol. 71, no. 5, pp. 306-314, 2004.

[10] C. Adrienne and C. Manigandan, "Inpatient occupational therapists hand-splinting practice for clients with stroke: A cross-sectional survey from ireland," Journal of neurosciences in rural practice, vol. 2, no. 2, p. 141, 2011.

[11] S.-K. Stavrakos, S. Ahmed-Kristensen, and T. Goldman, "Using archetypes to create user panels for usability studies: Streamlining focus groups and user studies," Applied ergonomics, vol. 56, pp. 108-116, 2016.

[12] S.-K. Stavrakos and S. Ahmed-Kristensen, "Assessment of anthropometric methods in headset design," in DS 70: Proceedings of DESIGN 2012, the 12th International Design Conference, Dubrovnik, Croatia, 2012.

[13] X. Tan, L. He, J. Cao, W. Chen, and T. Nanayakkara, "A soft pressure sensor skin for hand and wrist orthoses," IEEE Robotics and Automation Letters, vol. 5, no. 2, pp. 2192-2199, 2020.

[14] C. P. Witana, R. S. Goonetilleke, S. Xiong, and E. Y. Au, "Effects of surface characteristics on the plantar shape of feet and subjects' perceived sensations," Applied Ergonomics, vol. 40, no. 2, pp. 267-279, 2009.

[15] E. E. Fess and C. A. Philips, Hand splinting: principles and methods. Mosby Incorporated, 1987.

[16] M. A. Jacobs, N. M. Austin, et al., Orthotic intervention for the hand and upper extremity: Splinting principles and process. Lippincott Williams \& Wilkins, 2013. 
[17] A. Paterson, R. Bibb, and R. Campbell, "Evaluation of a digitised splinting approach with multiple-material functionality using additive manufacturing technologies," in Proceedings of the Solid Freeform Fabrication Symposium, vol. 2012, 2012, pp. 656-72.

[18] A. Stefanovich, C. Williams, P. McKee, E. Hagemann, and H. Carnahan, "Development and validation of tools for evaluation of orthosis fabrication," American Journal of Occupational Therapy, vol. 66, no. 6, pp. 739-746, 2012.

[19] K. Ghoseiri and H. Bahramian, "User satisfaction with orthotic and prosthetic devices and services of a single clinic," Disability and rehabilitation, vol. 34, no. 15, pp. 1328-1332, 2012.

[20] E. Hagemann et al., "Using model hands for learning orthotic fabrication," American Journal of Occupational Therapy, vol. 68, no. 1, pp. 86-94, 2014.

[21] M. Wong, J. Cheng, M. Wong, and S. So, "A work study of the cad/cam method and conventional manual method in the fabrication of spinal orthoses for patients with adolescent idiopathic scoliosis," Prosthetics and orthotics international, vol. 29, no. 1, pp. 93-105, 2005.

[22] A. A. Polliack, S. Elliot, C. Caves, D. R. McNeal, and S. E. Landsberger, "Lower extremity orthoses for children with myelomeningocele: user and orthotist perspectives," JPO: Journal of Prosthetics and Orthotics, vol. 13, no. 4, pp. 123-129, 2001.

[23] M. Richardson, G. Jones, and M. Torrance, "Identifying the task variables that influence perceived object assembly complexity," Ergonomics, vol. 47, no. 9, pp. 945-964, 2004.

[24] R. Q. Wolever, M. Jordan, K. Lawson, and M. Moore, "Advancing a new evidence-based professional in health care: job task analysis for health and wellness coaches," BMC health services research, vol. 16, no. 1, p. 205, 2016.

[25] V. A. Banks, N. A. Stanton, and C. Harvey, "What the drivers do and do not tell you: using verbal protocol analysis to investigate driver behaviour in emergency situations," Ergonomics, vol. 57, no. 3, pp. 332-342, 2014.

[26] J. Rose, C. Bearman, A. Naweed, and J. Dorrian, "Proceed with caution: using verbal protocol analysis to measure situation awareness," Ergonomics, vol. 62 , no. 1, pp. 115-127, 2019.

[27] S. Ahmed, K. M. Wallace, and L. T. Blessing, "Understanding the differences between how novice and experienced designers approach design tasks," Research in engineering design, vol. 14, no. 1, pp. 1-11, 2003.

[28] Y. Zhou, E. Cameron, G. Forbes, and G. Humphris, "Development of a novel coding scheme (sabics) to record nurse-child interactive behaviours in a community dental preventive intervention," Patient education and counseling, vol. 88, no. 2, pp. 268-276, 2012.

[29] X. Tan, W. Chen, J. Cao, and S. Ahmed-Kristensen. "IDENTIFY CRITICAL DATA DURING PRODUCT CUSTOMISATION-A CASE STUDY OF ORTHOSES FABRICATION.” In Proceedings of the Design Society: DESIGN Conference, vol. 1, pp. 413-422, Cambridge University Press, 2020.

[30] K. A. Ericsson and H. A. Simon, Protocol analysis: Verbal reports as data. the MIT Press, 1984.

[31] M. Bannert and C. Mengelkamp, "Assessment of metacognitive skills by means of instruction to think aloud and reflect when prompted. does the verbalisation method affect learning?" Metacognition and Learning, vol. 3, no. 1, pp. 39-58, 2008.

[32] H. Lundgren-Laine and S. Salantera, "Think-aloud technique and protocol analysis in clinical decision-making research," Qualitative health research, vol. 20 , no. 4, pp. 565-575, 2010.

[33] T. D. Wilson, "The proper protocol: Validity and completeness of verbal reports," Psychological Science, vol. 5, no. 5, pp. 249-252, 1994.

[34] M. T. Chi, "Quantifying qualitative analyses of verbal data: A practical guide," The journal of the learning sciences, vol. 6, no. 3, pp. 271-315, 1997.

[35] D. Narvaez, "Using discourse processing methods to study moral thinking," Educational Psychology Review, vol. 11, no. 4, pp. 377-393, 1999.

[36] R. Richard and R. S. Ward, "Splinting strategies and controversies," The Journal of burn care \& rehabilitation, vol. 26, no. 5, pp. 392-396, 2005.

[37] G. M. Jensen, K. F. Shepard, and L. M. Hack, "The novice versus the experienced clinician: insights into the work of the physical therapist," Physical Therapy, vol. 70, no. 5, pp. 314-323, 1990.

[38] R. Prosser and W. B. Conolly, "Complications following surgical treatment for dupuytren's contracture," Journal of Hand Therapy, vol. 9, no. 4, pp. 344-348, 1996. 